\title{
A class of functional equations associated with almost periodic functions ${ }^{1}$
}

\section{J.M. Sepulcre and T. Vidal}

Department of Mathematics, University of Alicante, 03080-Alicante, Spain.

ABSTRACT In this paper we will get a class of functional equations involving a countable set of terms, summed by the well known Bochner-Fejér summation procedure, which are closely associated with the set of almost periodic functions. We will show that the zeros of a prefixed almost periodic function determine analytic solutions of such a functional equation associated with it, and we will obtain other solutions which are analytic or meromorphic on a certain domain.

AMS Subject Classification: Primary: 30D05, 39B32, 42A75; Secondary: 39Bxx, 30Axx.

Key Words: Almost periodic functions; Dirichlet Series; Bochner-Fejér summation method; Zeros of Analytic Functions; Functional equations.

\section{Introduction}

The exponential sums of the form

$$
a_{1} e^{\lambda_{1} s}+\ldots+a_{j} e^{\lambda_{j} s}+\ldots, s \in \mathbb{C},
$$

where the coefficients $a_{1}, a_{2}, \ldots, a_{j}, \ldots$ are complex numbers and the frequencies $\lambda_{1}<\lambda_{2}<\ldots<\lambda_{j}<\ldots$ are pair-wise distinct real numbers, are connected with the theory of almost periodic functions of a complex variable, which was discussed in [4] (see also [3, 5, 6, 7, 8]). As in classical Fourier analysis, every almost periodic function is associated with a certain exponential series of the form (1.1), called its Dirichlet series, which determines the given almost periodic function (see [3, p.147], [6, p.77] or [8, p.312]).

Concerning the theory of almost periodic functions, we recall that if $f(s)$ is a function of a complex variable $s=\sigma+i t$ which is analytic in a vertical strip $U=\{s=\sigma+i t: \alpha<\sigma<\beta\}(-\infty \leq \alpha<\beta \leq \infty)$, then it is called almost periodic in $U$, and we denote it as $f \in A P(U, \mathbb{C})$, if for every $\varepsilon>0$ there exists a number $l=l(\varepsilon)>0$ such that any interval of length $l$ contains a number $\tau$ satisfying the inequality $|f(s+i \tau)-f(s)| \leq \varepsilon$ for $s$ in every reduced strip of the form $\left\{s=\sigma+i t: \sigma_{1} \leq \sigma \leq \sigma_{2}\right\}$ of $U$. So, the set of almost periodic functions $A P(U, \mathbb{C})$ coincides with the set of functions which can be approximated uniformly in every reduced strip by exponential polynomials of the form

$$
b_{1} e^{\lambda_{1} s}+\ldots+b_{n} e^{\lambda_{n} s}, s \in \mathbb{C}, n \geq 2,
$$

where $\left\{\lambda_{1}, \lambda_{2}, \ldots, \lambda_{n}\right\}$ is a set of distinct real numbers and $b_{j} \in \mathbb{C}$ for each $j=1,2, \ldots, n$ (see for example [6, Theorem 3.18]).

\footnotetext{
${ }^{1}$ This is a preprint of an article published in Aequationes mathematicae. The final authenticated version is available online at: https://doi.org/10.1007/s00010-020-00732-3
} 
It is convenient to remark that an exponential sum of the form (1.1) is not necessarily associated with a convergent series that defines a holomorphic function. However, even in the case that the sequence of the partial sums of the Dirichlet series of a function $f \in A P(U, \mathbb{C})$ does not converge uniformly, there exists a summation procedure, called the Bochner-Fejér's summation, which assures the existence of a sequence of exponential polynomials of the type $P_{k}(s)=\sum_{j>1} p_{j, k} a_{j} e^{\lambda_{j} s}$, where for each $k$ only a finite number of the factors $p_{j, k}$ differ from zero, converging uniformly to $f$ in every reduced strip in $U$ and converging formally to its Dirichlet series on $U$ [3, Polynomial approximation theorem, pgs. 50,148]. Plainly, the formal convergence implies that $\lim _{k \rightarrow \infty} p_{j, k}=1$ for each $j \geq 1$.

On the other hand, in this paper we will also handle expressions of the type

$$
a_{1} f\left(\gamma_{1} s\right)+a_{2} f\left(\gamma_{2} s\right)+\ldots+a_{j} f\left(\gamma_{j} s\right)+\ldots=0, s \in S,
$$

where $a_{j}$ are complex numbers, $\gamma_{j}$ constitute a countable set of pair-wise distinct numbers and $f(s)$ is a complex function in $s$ whose domain includes the points $\gamma_{j} s$, with $s$ in a certain set $S$. We shall refer to these expressions as functional series equations or simply functional equations. This process requires functional series in the left member of (1.3) which are summable on $S$ by some prefixed summation method (not necessarily that consisting of the limit of its partial sums). In the context of this paper, it must be kept in mind that we will use the Bochner-Fejér's summation method (see Definition 2.1).

Given $n \geq 2$ an integer number, a particular case of the functional equations of type (1.3) is given by

$$
b_{1} f\left(\gamma_{1} s\right)+b_{2} f\left(\gamma_{2} s\right)+\ldots+b_{n} f\left(\gamma_{n} s\right)=0, s \in S,
$$

where $b_{j}$ are complex numbers and $\left\{\gamma_{1}, \gamma_{2}, \ldots, \gamma_{n}\right\}$ is a finite set of pair-wise distinct positive numbers. When $S=\mathbb{C} \backslash\{0\}$, we know that a functional equation of the form (1.4) is associated with the exponential polynomial

$$
P_{n}(s)=b_{1} e^{s \log \gamma_{1}}+\ldots+b_{n} e^{s \log \gamma_{n}},
$$

which is of type (1.2). Indeed, it is clear that the functions of the form $f_{n, j}(s)=$ $e^{\alpha_{n, j} \log s}$, where $\alpha_{n, j}$ is a zero of $P_{n}(s)$ and $\log s$ denotes the principal branch of the logarithm of $s \neq 0$, are solutions analytic on $\mathbb{C} \backslash(-\infty, 0]$ of the functional equation (1.4) (see also [12]). It is worth noting that this connection was previously shown for the special cases

$$
f(s)+f(2 s)+\ldots+f(n s)=0, n \in \mathbb{N}, n \geq 2, s \in \mathbb{C} \backslash\{0\},
$$

which were proposed by Mora in order to model certain processes related to combustion of hydrogen in a car engine for small values of $n$ (see [10, p. 466]), and which lead to a strong connection with the partial sums of the Riemann zeta function $\zeta_{n}(s)=1+2^{-s}+\ldots+n^{-s}, n \geq 2$ (see $\left.[10,11]\right)$. Another particular case of functional equations of type (1.4) was studied by Almira and Abu-Helaiel (see [1]). 
The main purpose in this paper is to extend this previous connection to the more general case of almost periodic functions (not only exponential polynomials). Specifically, we will show that the zeros of a prefixed almost periodic function determine solutions analytic on $\mathbb{C} \backslash(-\infty, 0]$ of a concrete functional equation of type (1.3) associated with such a function (see Proposition 2.4). More generally, this process can be extended to find solutions analytic on every set $\mathbb{C} \backslash R_{\theta}$, where $R_{\theta}$ is the ray $\left\{r e^{i \theta}: r \geq 0\right\}, \theta \in(-2 \pi, 0]$ (see Proposition 2.9). Moreover, we will obtain a vector space of real continuous solutions of such a functional equation for the case that the parameter $s$ varies in the set of positive real numbers (see Corollary 2.7).

Concurrently with the development above, another purpose in this paper is to show that any solution of a functional equation of type (1.4), where $S \subset \mathbb{C}$ is a certain set that contains 0 , cannot be analytic at the origin, except for the solutions given by polynomials (see Proposition 3.1 ). This also extends [12, Proposition 5], which showed that, under the condition of positive coefficients $b_{j}>0$, the only solution of such a functional equation that is analytic at 0 is the trivial solution $f \equiv 0$. Moreover, under the hypothesis of analyticity on an annulus $S$ centered at 0 , we shall obtain the form of other solutions of such functional equations which are analytic on $\mathbb{C} \backslash\{0\}$ (see Proposition 3.4). In fact, we will also prove that the meromorphic functions whose Laurent expansion about 0 has a finite number of nonzero terms are solutions of infinitely many functional equations of type (1.4) where $S$ is an arbitrary annulus centered at 0 (see Proposition 3.7).

\section{The practical correspondence between almost periodic functions and their underlying func- tional equations}

Given a set $\left\{\gamma_{1}, \gamma_{2}, \ldots\right\}$ of infinitely many distinct real numbers and $a_{j} \in \mathbb{C}$ for $j=1,2, \ldots$, in this section we will focus our attention on the group of functional equations given by

$$
a_{1} f\left(\gamma_{1} s\right)+a_{2} f\left(\gamma_{2} s\right)+\ldots+a_{j} f\left(\gamma_{j} s\right)+\ldots=0, \text { with } s \in S,
$$

where $f(s)$ is a complex function whose domain includes the points $\gamma_{j} s, j=$ $1,2, \ldots$, with $s$ in a certain set $S \subset \mathbb{C}$ satisfying that the functional series in the left member is summable on $S$ by some prefixed summation method. In particular, consider the following definition.

Definition 2.1 Given a set $\Gamma=\left\{\gamma_{1}, \gamma_{2}, \ldots\right\}$ of infinitely many distinct real numbers, and $k \in \mathbb{N}$, by following the same procedure as described in [3, pp. $47,48]$, consider the set of multiples $\left\{p_{1, k}, p_{2, k}, \ldots, p_{m_{k}, k}\right\}$, with $m_{k}=\max \{j \geq$ $\left.1: p_{j, k} \neq 0\right\}$, which are real numbers obtained from $\Gamma$, a basis for $\Gamma$ (called basic numbers in [3]) and $k$. If $a_{j} \in \mathbb{C}$ for $j=1,2, \ldots$, we will say that the functional 
series $a_{1} f\left(\gamma_{1} s\right)+a_{2} f\left(\gamma_{2} s\right)+\ldots+a_{j} f\left(\gamma_{j} s\right)+\ldots$ is (Bochner-Fejér) summable on $S$ when there exists

$$
\lim _{k \rightarrow \infty} p_{1, k} a_{1} f\left(\gamma_{1} s\right)+p_{2, k} a_{2} f\left(\gamma_{2} s\right)+\ldots+p_{m_{k}, k} a_{m_{k}} f\left(\gamma_{m_{k}} s\right)
$$

for any $s \in S$.

Under this definition, we will say that a complex function $g$ is a non-trivial solution of (1.1) when $g$ is not the null function and $a_{1} g\left(\gamma_{1} s\right)+a_{2} g\left(\gamma_{2} s\right)+\ldots+$ $a_{j} g\left(\gamma_{j} s\right)+\ldots=0$ for all $s \in S$ in the sense that the functional series in the left member is (Bochner-Fejér) summable on $S$ and it is equal to 0 for all $s \in S$.

On the other hand, inspired by the Bochner-Fejér summation method, we will also consider the following classes of functions included in the spaces of almost periodic functions $A P(U, \mathbb{C})$. We recall that the elements of these spaces also correspond to the so-called uniformly almost periodic functions which were used in [3].

Definition 2.2 Let $\Lambda=\left\{\lambda_{1}, \lambda_{2}, \ldots, \lambda_{j}, \ldots\right\}$ be a countable set of distinct real numbers. We will say that a function $g: U \subset \mathbb{C} \rightarrow \mathbb{C}$ is in the class $\mathcal{D}_{\Lambda, U}$ if it is an almost periodic function in $A P(U, \mathbb{C})$ whose associated Dirichlet series is of the form

$$
\sum_{j \geq 1} a_{j} e^{\lambda_{j} s}, a_{j} \in \mathbb{C}, \lambda_{j} \in \Lambda
$$

where $U$ is a strip of the type $\{s \in \mathbb{C}: \alpha<\operatorname{Re} s<\beta\}$, with $-\infty \leq \alpha<\beta \leq \infty$.

As it was said in the introduction, given $g(s) \in A P(U, \mathbb{C})$, there exists a sequence of finite exponential sums of the form $P_{k}(s)=\sum_{j \geq 1} p_{j, k} a_{j} e^{\lambda_{j} s}$, $k=1,2, \ldots$, called Bochner-Fejér polynomials, which converges uniformly to $g$ in every reduced strip in $U$ and converges formally to the Dirichlet series on $U$. In fact, for each $k$, only a finite number of the terms $p_{j, k}$ differ from zero (and $\left\{p_{j, k}\right\}_{k \geq 1}$ tends to 1 as $k$ goes to infinity), and they depend on the real frequencies $\lambda_{j}$ but not on the values of the coefficients $a_{j}[3, \mathrm{p} .48]$. This fact is key to proving the first results of this paper.

The following lemma shows a particular case for which our functional series are summable according to definition 2.1.

Lemma 2.3 Let $\sum_{n>1} a_{n} e^{\lambda_{n} s}$ be the Dirichlet series, of the form (2.1), of a certain almost periodic function in a class $\mathcal{D}_{\Lambda, U}$, with $\Lambda=\left\{\lambda_{1}, \lambda_{2}, \ldots, \lambda_{j}, \ldots\right\}$. If $f_{w}(s)=e^{w \log s}$, with $w \in U$, then the functional series

$$
a_{1} f_{w}\left(e^{\lambda_{1}} s\right)+a_{2} f_{w}\left(e^{\lambda_{2}} s\right)+\ldots+a_{j} f_{w}\left(e^{\lambda_{j}} s\right)+\ldots
$$

is (Bochner-Fejér) summable on $\mathbb{C} \backslash\{0\}$.

Proof. Let $P_{k}(s)=\sum_{j \geq 1} p_{j, k} a_{j} e^{\lambda_{j} s}, k=1,2, \ldots$, be a sequence of BochnerFejér polynomials uniformly convergent to a function $g(s) \in A P(U, \mathbb{C})$ and take $m_{k}:=\max \left\{j \geq 1: p_{j, k} \neq 0\right\}, k=1,2, \ldots$. Given $w \in U$, consider the function 
$f_{w}(s):=e^{w \log s}, s \in \mathbb{C} \backslash\{0\}$. Since $\log \left(e^{\lambda_{j}} s\right)=\lambda_{j}+\log s$ for each $j \geq 1$ and any $s \in \mathbb{C} \backslash\{0\}$, it is clear that

$$
\begin{gathered}
\lim _{k \rightarrow \infty} p_{1, k} a_{1} f_{w}\left(e^{\lambda_{1}} s\right)+p_{2, k} a_{2} f_{w}\left(e^{\lambda_{2}} s\right)+\ldots+p_{m_{k}, k} a_{m_{k}} f_{w}\left(e^{\lambda_{m_{k}} s}\right)= \\
\lim _{k \rightarrow \infty} e^{w \log s}\left(p_{1, k} a_{1} e^{\lambda_{1} w}+p_{2, k} a_{2} e^{\lambda_{2} w}+\ldots+p_{m_{k}, k} a_{m_{k}} e^{\lambda_{m_{k}} w}\right)= \\
\lim _{k \rightarrow \infty} e^{w \log s} P_{k}(w)=e^{w \log s} g(w)
\end{gathered}
$$

for any $s \in \mathbb{C} \backslash\{0\}$, which proves the result.

Given an almost periodic function $g(s) \in A P(U, \mathbb{C})$, we next establish a connection of it with a concrete functional equation which presents analytic solutions obtained from the zeros of $g(s)$. This extends a result already proved in [12, Corollary 3].

Proposition 2.4 Let $g(s)$ be an almost periodic function in a class $\mathcal{D}_{\Lambda, U}$ whose Dirichlet series, $\sum_{n \geq 1} a_{n} e^{\lambda_{n} s}$, is of the form (2.1), with $\Lambda=\left\{\lambda_{1}, \lambda_{2}, \ldots, \lambda_{j}, \ldots\right\}$. Then the functions $f_{w_{0}}(s)=e^{w_{0} \log s}$, with $w_{0}$ belonging to the set of the zeros of $g(s)$, are solutions analytic on $\Omega=\mathbb{C} \backslash(-\infty, 0]$ of the functional equation

$$
a_{1} f\left(e^{\lambda_{1}} s\right)+a_{2} f\left(e^{\lambda_{2}} s\right)+\ldots+a_{j} f\left(e^{\lambda_{j}} s\right)+\ldots=0, s \in \mathbb{C} \backslash\{0\} .
$$

Proof. Let $P_{k}(s)=\sum_{j \geq 1} p_{j, k} a_{j} e^{\lambda_{j} s}, k=1,2, \ldots$, be a sequence of BochnerFejér polynomials uniformly convergent to the function $g(s) \in A P(U, \mathbb{C})$ and take $m_{k}:=\max \left\{j \geq 1: p_{j, k} \neq 0\right\}, k=1,2, \ldots$. Now, let $w_{0}$ be a zero of $g(s)$, and consider the function $f_{w_{0}}(s):=e^{w_{0} \log s}, s \in \mathbb{C} \backslash\{0\}$. By Lemma 2.3, we have that $a_{1} f_{w_{0}}\left(e^{\lambda_{1}} s\right)+a_{2} f_{w_{0}}\left(e^{\lambda_{2}} s\right)+\ldots+a_{j} f_{w_{0}}\left(e^{\lambda_{j}} s\right)+\ldots$ is summable on $\mathbb{C} \backslash\{0\}$ and, in fact, we have that

$$
\begin{aligned}
\lim _{k \rightarrow \infty} p_{1, k} a_{1} f_{w_{0}}\left(e^{\lambda_{1}} s\right)+p_{2, k} a_{2} f_{w_{0}}\left(e^{\lambda_{2}} s\right)+\ldots+p_{m_{k}, k} a_{m_{k}} f_{w_{0}}\left(e^{\lambda_{m_{k}}} s\right) & = \\
\lim _{k \rightarrow \infty} e^{w_{0} \log s}\left(p_{1, k} a_{1} e^{\lambda_{1} w_{0}}+p_{2, k} a_{2} e^{\lambda_{2} w_{0}}+\ldots+p_{m_{k}, k} a_{m_{k}} e^{\lambda_{m_{k}} w_{0}}\right) & = \\
\lim _{k \rightarrow \infty} e^{w_{0} \log s} P_{k}\left(w_{0}\right)=e^{w_{0} \log s} g\left(w_{0}\right) & =0
\end{aligned}
$$

for any $s \in \mathbb{C} \backslash\{0\}$, which proves the result.

Inspired by the previous result, we next introduce the following definition.

Definition 2.5 Let $g(s)$ be an almost periodic function in $A P(U, \mathbb{C})$ whose Dirichlet series is of the form $\sum_{n \geq 1} a_{n} e^{\lambda_{n} s}$, with $\Lambda=\left\{\lambda_{1}, \lambda_{2}, \ldots, \lambda_{j}, \ldots\right\} a$ countable set of distinct ordered real numbers, and $a_{j} \in \mathbb{C}$ for each $j=1,2, \ldots$. Then the functional equation

$$
a_{1} f\left(e^{\lambda_{1}} s\right)+a_{2} f\left(e^{\lambda_{2}} s\right)+\ldots+a_{j} f\left(e^{\lambda_{j}} s\right)+\ldots=0, s \in \mathbb{C} \backslash\{0\},
$$

will be called the functional equation associated with $g(s)$.

In the space of functions analytic on $\Omega=\mathbb{C} \backslash(-\infty, 0]$, let $D$ be the linear operator defined by $D(f)=s f^{\prime}$, where $f^{\prime}(s)$ is the derivative of $f(s)$. Concerning this operator, we next prove the following result. 
Proposition 2.6 Let $g(s)$ be an almost periodic function in a class $\mathcal{D}_{\Lambda, U}$ whose Dirichlet series, $\sum_{n>1} a_{n} e^{\lambda_{n} s}$, is of the form (2.1), with $\Lambda=\left\{\lambda_{1}, \lambda_{2}, \ldots, \lambda_{j}, \ldots\right\}$. In the space of functions analytic on $\Omega$, the proper vectors of the operator $D$ are solutions analytic on $\Omega$ of the functional equation (2.2) associated with $g(s)$ if and only if the corresponding proper values of $D$ are the zeros of $g(s)$.

Proof. Let $f_{w_{0}}$ be a proper vector of $D$ corresponding to a generic proper value $w_{0} \in U$. Then $D\left(f_{w_{0}}\right)=w_{0} f_{w_{0}}$ or, equivalently, $f_{w_{0}}$ satisfies the equation $s f_{w_{0}}^{\prime}=w_{0} f_{w_{0}}$, whose solution analytic on $\Omega$ is $f_{w_{0}}(s)=K e^{w_{0} \log s}$, with $K$ an arbitrary constant. By Lemma 2.3, we have that $a_{1} f_{w_{0}}\left(e^{\lambda_{1}} s\right)+a_{2} f_{w_{0}}\left(e^{\lambda_{2}} s\right)+$ $\ldots+a_{j} f_{w_{0}}\left(e^{\lambda_{j}} s\right)+\ldots$ is summable on $\mathbb{C} \backslash\{0\}$. Now, let $P_{k}(s)=\sum_{j>1} p_{j, k} a_{j} e^{\lambda_{j} s}$, $k=1,2, \ldots$, be a sequence of Bochner-Fejér polynomials uniformly convergent to the function $g(s) \in A P(U, \mathbb{C})$ and take $m_{k}:=\max \left\{j \geq 1: p_{j, k} \neq 0\right\}$, $k=1,2, \ldots$. Since $\log \left(e^{\lambda_{j}} s\right)=\lambda_{j}+\log s$ for each $j \geq 1$ and any $s \in \mathbb{C} \backslash\{0\}$, we have

$$
\begin{aligned}
& p_{1, k} a_{1} f_{w_{0}}\left(e^{\lambda_{1}} s\right)+p_{2, k} a_{2} f_{w_{0}}\left(e^{\lambda_{2}} s\right)+\ldots+p_{m_{k}, k} a_{m_{k}} f_{w_{0}}\left(e^{\lambda_{m_{k}} s}\right)= \\
& K e^{w_{0} \log s}\left(p_{1, k} a_{1} e^{\lambda_{1} w_{0}}+p_{2, k} a_{2} e^{\lambda_{2} w_{0}}+\ldots+p_{m_{k}, k} a_{m_{k}} e^{\lambda_{m_{k}} w_{0}}\right),
\end{aligned}
$$

for every $k \geq 1$. Hence, $f_{w_{0}}(s)$ is a solution of $(2.2)$ if and only if $\lim _{k \rightarrow \infty} P_{k}\left(w_{0}\right)=0$ or, equivalently, $g\left(w_{0}\right)=0$.

Particularly, in connection with (2.2) for $s=x>0$, a solution of the functional equation

$$
a_{1} f\left(e^{\lambda_{1}} x\right)+a_{2} f\left(e^{\lambda_{2}} x\right)+\ldots+a_{j} f\left(e^{\lambda_{j}} x\right)+\ldots=0, x>0,
$$

is given by $f_{w_{0}}(x)=x^{w_{0}}$, with $w_{0}$ belonging to the set of the zeros of the function $g(s)$. If $w_{0}=\sigma_{0}+i t_{0}$, then $f_{w_{0}}(x)=x^{\sigma_{0}} e^{i t_{0} \log x}$, and we obtain real solutions of (2.3) of the form

$$
x^{\sigma_{0}}\left(c \cos \left(t_{0} \log x\right)+d \sin \left(t_{0} \log x\right)\right), c, d \in \mathbb{R} .
$$

Consequently, we get the following important result which improves [12, Corollary 4].

Corollary 2.7 Let $g(s)$ be an almost periodic function in a class $\mathcal{D}_{\Lambda, U}$ whose Dirichlet series, $\sum_{n \geq 1} a_{n} e^{\lambda_{n} s}$, is of the form (2.1), with $\Lambda=\left\{\lambda_{1}, \lambda_{2}, \ldots, \lambda_{j}, \ldots\right\}$. Then every zero of $g(s)$ generates a vector space of real continuous solutions of the functional equation (2.3).

Furthermore, an easy consequence of the previous corollary is that every real continuous solution $f(x)$ of $(2.3)$ defines a function

$$
h(x)=\left\{\begin{array}{cc}
f(x), & \text { if } x>0 \\
0, & \text { if } x=0 \\
f(-x), & \text { if } x<0
\end{array}\right.
$$


which is a continuous solution, except possibly at the point 0 , of the functional equation

$$
a_{1} f\left(e^{\lambda_{1}} x\right)+a_{2} f\left(e^{\lambda_{2}} x\right)+\ldots+a_{j} f\left(e^{\lambda_{j}} x\right)+\ldots=0, x \in \mathbb{R} .
$$

We next prove that the functions $f_{w_{0}}(s)=e^{w_{0} \log s}$, with $w_{0}$ belonging to the set of zeros of $g(s)$, are linearly independent in the vector space of functions analytic on $\Omega$. The proof is similar to that of [12, Proposition 6].

Proposition 2.8 Let $g(s)$ be an almost periodic function in a class $\mathcal{D}_{\Lambda, U}$ whose Dirichlet series is of the form (2.1). Then the functions $f_{w_{0}}(s)=e^{w_{0} \log s}$, with $w_{0}$ belonging to the set of the zeros of $g(s)$, which are solutions analytic on $\Omega=\mathbb{C} \backslash(-\infty, 0]$ of the functional equation (2.2) associated with $g(s)$, are linearly independent in the vector space of functions analytic on $\Omega$.

Proof. It was proved in Proposition 2.4 that the functions $f_{w_{0}}(s)=e^{w_{0} \log s}$, with $w_{0}$ belonging to the set of the zeros of $g(s)$, are solutions analytic on the set $\Omega=\mathbb{C} \backslash(-\infty, 0]$. Let $w_{1}, w_{2}, \ldots, w_{m}$ be $m$ distinct zeros of $g(s)$ with $w_{j}=\sigma_{j}+i t_{j}, j=1,2, \ldots, m$, and let $t:=\max \left\{\left|t_{j}\right|: j=1,2, \ldots, m\right\} \geq 0$. Also, we can determine $y>1$ such that $t \log y<\pi$. Then $-\pi<t_{j} \log y<\pi$, $j=1,2, \ldots, m$, and therefore the principal argument of $y^{w_{j}}$ is $t_{j} \log y$ for each $j=1,2, \ldots, m$. Now, consider the numbers $c_{j}:=y^{j-1}, j=1,2, \ldots, m$, and let $f_{w_{1}}, f_{w_{2}}, \ldots, f_{w_{m}}$ denote the power functions $e^{w_{1} \log s}, e^{w_{2} \log s}, \ldots, e^{w_{m} \log s}$ respectively. Thus the determinant

$$
D=\left|\begin{array}{cccc}
f_{w_{1}}\left(c_{1}\right) & f_{w_{2}}\left(c_{1}\right) & \cdots & f_{w_{m}}\left(c_{1}\right) \\
f_{w_{1}}\left(c_{2}\right) & f_{w_{2}}\left(c_{2}\right) & \cdots & f_{w_{m}}\left(c_{2}\right) \\
\vdots & \vdots & \ddots & \vdots \\
f_{w_{1}}\left(c_{m}\right) & f_{w_{2}}\left(c_{m}\right) & \cdots & f_{w_{m}}\left(c_{m}\right)
\end{array}\right|=\left|\begin{array}{cccc}
1 & 1 & \cdots & 1 \\
d_{1} & d_{2} & \cdots & d_{m} \\
\vdots & \vdots & \ddots & \vdots \\
d_{1}^{m-1} & d_{2}^{m-1} & \cdots & d_{m}^{m-1}
\end{array}\right|,
$$

where $d_{j}:=y^{w_{j}}, j=1,2, \ldots, m$, is of Vandermonde type and so

$$
D=\prod_{k>j(k, j=1, \ldots, m)}\left(d_{k}-d_{j}\right) .
$$

Since $d_{j}$ are all distinct because they have different arguments or absolute values, we deduce that $D \neq 0$. This means that, for the $m$ values $c_{1}, c_{2}, \ldots, c_{m}$, the system

$$
\begin{aligned}
x_{1} f_{w_{1}}\left(c_{1}\right)+x_{2} f_{w_{2}}\left(c_{1}\right)+\ldots+x_{m} f_{w_{m}}\left(c_{1}\right) & =0 \\
x_{1} f_{w_{1}}\left(c_{2}\right)+x_{2} f_{w_{2}}\left(c_{2}\right)+\ldots+x_{m} f_{w_{m}}\left(c_{2}\right) & =0 \\
\ldots & =0 \\
x_{1} f_{w_{1}}\left(c_{m}\right)+x_{2} f_{w_{2}}\left(c_{m}\right)+\ldots+x_{m} f_{w_{m}}\left(c_{m}\right) & =0
\end{aligned}
$$

has only the trivial solution $x_{1}=x_{2}=\ldots=x_{m}=0$. Hence the expression $x_{1} f_{w_{1}}(s)+x_{2} f_{w_{2}}(s)+\ldots+x_{m} f_{w_{m}}(s)=0$ implies $x_{1}=x_{2}=\ldots=x_{m}=0$, i.e. the functions $f_{w_{1}}(s), f_{w_{2}}(s), \ldots, f_{w_{m}}(s)$ are linearly independent. 
Now, given $\theta \in \mathbb{R}$, let $\log _{\theta} s$ denote the branch of the logarithm function such that the argument of $s$ is in $[\theta, \theta+2 \pi)$, that is $\log _{\theta} s=\log |s|+i \arg _{\theta} s$, with $\arg _{\theta}$ the unique argument of $s$ in $[\theta, \theta+2 \pi)$. Recall that the function $\log _{\theta} s$ is analytic on $\mathbb{C} \backslash R_{\theta}$, where $R_{\theta}$ is the ray $\left\{r e^{i \theta}: r \geq 0\right\}$. Proposition 2.4 can be now generalised in the following sense.

Proposition 2.9 Let $g(s)$ be an almost periodic function in a class $\mathcal{D}_{\Lambda, U}$ whose Dirichlet series, $\sum_{n>1} a_{n} e^{\lambda_{n} s}$, is of the form (2.1), with $\Lambda=\left\{\lambda_{1}, \lambda_{2}, \ldots, \lambda_{j}, \ldots\right\}$. Given $\theta \in(-2 \pi, 0]$, the functions $f_{w_{0}}(s)=e^{w_{0} \log _{\theta} s}$, with $w_{0}$ belonging to the set of the zeros of $g(s)$, are solutions analytic on $\mathbb{C} \backslash R_{\theta}$ of the functional equation (2.2) associated with $g(s)$.

Proof. Given $g(s) \in \mathcal{D}_{\Lambda, U}$ and $\theta \in(-2 \pi, 0]$, take $f_{w_{0}}(s)=e^{w_{0} \log _{\theta} s}$ with $w_{0}$ belonging to the set of the zeros of $g(s)$. Since $\log _{\theta}\left(e^{\lambda_{j}} s\right)=\lambda_{j}+\log _{\theta} s$ for each $j \geq 1$ and any $s \in \mathbb{C} \backslash\{0\}$, as in Lemma 2.3, we have that $a_{1} f_{w_{0}}\left(e^{\lambda_{1}} s\right)+$ $a_{2} f_{w_{0}}\left(e^{\lambda_{2}} s\right)+\ldots+a_{j} f_{w_{0}}\left(e^{\lambda_{j}} s\right)+\ldots$ is summable on $\mathbb{C} \backslash R_{\theta}$ and

$$
\begin{array}{r}
\lim _{k \rightarrow \infty} p_{1, k} a_{1} f_{w_{0}}\left(e^{\lambda_{1}} s\right)+p_{2, k} a_{2} f_{w_{0}}\left(e^{\lambda_{2}} s\right)+\ldots+p_{m_{k}, k} a_{m_{k}} f_{w_{0}}\left(e^{\lambda_{m_{k}}} s\right) \\
\lim _{k \rightarrow \infty} e^{w_{0} \log _{\theta} s}\left(p_{1, k} a_{1} e^{\lambda_{1} w_{0}}+p_{2, k} a_{2} e^{\lambda_{2} w_{0}}+\ldots+p_{m_{k}, k} a_{m_{k}} e^{\lambda_{m_{k}} w_{0}}\right)= \\
\lim _{k \rightarrow \infty} e^{w_{0} \log _{\theta} s} P_{k}\left(w_{0}\right)=e^{w_{0} \log _{\theta} s} g\left(w_{0}\right)=0
\end{array}
$$

for any $s \in \mathbb{C} \backslash R_{\theta}$.

\section{Solutions of the functional equations associ- ated with exponential polynomials}

Given an exponential polynomial $P(s)=a_{1} e^{\lambda_{1} s}+\ldots+a_{n} e^{\lambda_{n} s}, s \in \mathbb{C}, n \geq 2$, with $a_{j} \in \mathbb{C}$ and $\left\{\lambda_{1}, \ldots, \lambda_{n}\right\}$ a set of distinct real numbers, Proposition 2.9 shows that the functions of the form $f_{w_{0}}(s)=e^{w_{0} \log _{\theta} s}$, with $w_{0}$ a zero of $P(s)$, are solutions analytic on $\mathbb{C} \backslash R_{\theta}$ of the functional equation

$$
a_{1} f\left(e^{\lambda_{1}} s\right)+a_{2} f\left(e^{\lambda_{2}} s\right)+\ldots+a_{n} f\left(e^{\lambda_{n}} s\right)=0, s \in \mathbb{C} \backslash\{0\},
$$

associated with $P(s)$. In this respect, in this section we will handle functional equations of the type

$$
a_{1} f\left(\gamma_{1} s\right)+a_{2} f\left(\gamma_{2} s\right)+\ldots+a_{n} f\left(\gamma_{n} s\right)=0, s \in S,
$$

where $n \in \mathbb{N}, n \geq 2, a_{j}$ are complex numbers, $\gamma_{j}$ are pair-wise distinct positive numbers and $S$ is a certain set in $\mathbb{C}$. Hence the member on the left is certainly summable, and the expressions of the form (3.1) are included in this type of functional equations, which means that we will handle a particular case of the functional equations of type (1.3) which was already considered in [12]. In fact, we will show more solutions of the functional equation (3.1) (extended to the 
whole $\mathbb{C}$ or with other domains) which are not of the form $f_{w_{0}}(s)$ indicated above. We first show that any solution of such a functional equation cannot be analytic at the origin, except for the solutions given by polynomials.

Proposition 3.1 Let $f(s)$ be a complex solution of the functional equation

$$
a_{1} f\left(\gamma_{1} s\right)+a_{2} f\left(\gamma_{2} s\right)+\ldots+a_{n} f\left(\gamma_{n} s\right)=0, s \in S
$$

where $n \in \mathbb{N}, n \geq 2,\left\{\gamma_{1}, \gamma_{2}, \ldots, \gamma_{n}\right\}$ is a set of distinct positive numbers, $a_{j} \in \mathbb{C} \backslash\{0\}$ for each $j=1,2, \ldots, n$ and $S$ is a certain open set that contains 0 . If $f(s)$ is analytic at 0 , then it is a polynomial of, at most, $n-1$ terms.

Proof. Assume the existence of a solution of (3.2) which is analytic at 0, say $f(s)$. This means that $f(s)$ is analytic on an open set $D$ that contains 0 and $f(s)=\sum_{k>0} \frac{f^{(k)}(0)}{k !} s^{n} \forall s \in D$. Hence

$$
\begin{gathered}
a_{1} f\left(\gamma_{1} s\right)+a_{2} f\left(\gamma_{2} s\right)+\ldots+a_{n} f\left(\gamma_{n} s\right)= \\
a_{1} \sum_{k \geq 0} \frac{f^{(k)}(0)}{k !} \gamma_{1}^{k} s^{k}+a_{2} \sum_{k \geq 0} \frac{f^{(k)}(0)}{k !} \gamma_{2}^{k} s^{k}+\ldots+a_{n} \sum_{k \geq 0} \frac{f^{(k)}(0)}{k !} \gamma_{n}^{k} s^{k}= \\
\left(a_{1} f(0)+a_{2} f(0)+\ldots+a_{n} f(0)\right)+\left(a_{1} f^{\prime}(0) \gamma_{1}+a_{2} f^{\prime}(0) \gamma_{2}+\ldots+a_{n} f^{\prime}(0) \gamma_{n}\right) s+ \\
+\ldots+\left(a_{1} f^{(n-1)}(0) \gamma_{1}^{(n-1)}+a_{2} f^{(n-1)}(0) \gamma_{2}^{(n-1)}+\ldots+a_{n} f^{(n-1)}(0) \gamma_{n}^{(n-1)}\right) s^{n-1}+ \\
+\ldots=0,
\end{gathered}
$$

for all $s$ in an open set that contains 0 and is included in $S$. This yields that

$$
\begin{aligned}
f(0)\left(a_{1}+a_{2}+\ldots+a_{n}\right) & =0 \\
f^{\prime}(0)\left(a_{1} \gamma_{1}+a_{2} \gamma_{2}+\ldots+a_{n} \gamma_{n}\right) & =0 \\
\ldots & =0 \\
f^{(n-1)}(0)\left(a_{1} \gamma_{1}^{(n-1)}+a_{2} \gamma_{2}^{(n-1)}+\ldots+a_{n} \gamma_{n}^{(n-1)}\right) & =0 \\
\ldots= & 0 .
\end{aligned}
$$

If we took $\mathbf{a}=\left(a_{1}, a_{2}, \ldots\right)^{t}$ as the vector of the unknowns, this would lead to a matrix equation of the form $M \mathbf{a}=\mathbf{0}$, where

$$
M=\left(\begin{array}{cccc}
f(0) & f(0) & \cdots & f(0) \\
f^{\prime}(0) \gamma_{1} & f^{\prime}(0) \gamma_{2} & \cdots & f^{\prime}(0) \gamma_{n} \\
\vdots & \cdots & \ddots & \vdots \\
f^{(n-1)}(0) \gamma_{1}^{(n-1)} & f^{(n-1)}(0) \gamma_{2}^{(n-1)} & \cdots & f^{(n-1)}(0) \gamma_{n}^{(n-1)} \\
\vdots & \vdots & \vdots & \vdots
\end{array}\right)
$$

Under the hypothesis of the existence of at least $n$ values of the set given by $\left\{f(0), f^{\prime}(0), \ldots, f^{(n-1)}(0), \ldots\right\}$ which are nonzero, it would be satisfied that rank $M=n$ because of the fact that these $n$ rows of the matrix $M$ (associated 
with the values $f^{\left(\alpha_{1}\right)}(0), f^{\left(\alpha_{2}\right)}(0), \ldots, f^{\left(\alpha_{n}\right)}(0)$ different from zero) would give rise to a generalized Vandermonde determinant of the form

$$
f^{\left(\alpha_{1}\right)}(0) f^{\left(\alpha_{2}\right)}(0) \cdots f^{\left(\alpha_{n}\right)}(0)\left|\begin{array}{cccc}
d_{1}^{\alpha_{1}} & d_{2}^{\alpha_{1}} & \cdots & d_{n}^{\alpha_{1}} \\
d_{1}^{\alpha_{2}} & d_{2}^{\alpha_{2}} & \cdots & d_{n}^{\alpha_{2}} \\
\vdots & \vdots & \ddots & \vdots \\
d_{1}^{\alpha_{n}} & d_{2}^{\alpha_{n}} & \cdots & d_{n}^{\alpha_{n}}
\end{array}\right| \neq 0
$$

where $d_{j}:=\gamma_{j}, j=1,2, \ldots, n$, and $\alpha_{1}<\alpha_{2}<\ldots<\alpha_{n}$ (see for example [13, Theorem 1]). Equivalently, the trivial solution $a_{1}=a_{2}=\ldots=a_{n}=0$ would be the only solution of this system, which is a contradiction. Consequently, $f(s)$ is a polynomial of, at most, $n-1$ terms.

Remark 3.2 From the proof of the proposition above it is also deduced that if $P(s)=b_{1} s^{\alpha_{1}}+b_{2} s^{\alpha_{2}}+\ldots+b_{n-1} s^{\alpha_{n-1}}$, with $0 \leq \alpha_{1}<\alpha_{2}<\ldots<\alpha_{n-1}$ and $b_{j} \in \mathbb{C} \backslash\{0\}$ for each $j=1, \ldots, n-1$, is a polynomial of $n-1$ terms which is a solution of the functional equation (3.2), then any solution of such an equation is of the form

$$
f(s)=c_{1} s^{\alpha_{1}}+c_{2} s^{\alpha_{2}}+\ldots+c_{n-1} s^{\alpha_{n-1}},
$$

with $c_{1}, c_{2}, \ldots, c_{n-1} \in \mathbb{C}$. Indeed, $a_{1}, a_{2}, \ldots, a_{n}$ satisfy the system

$$
\begin{aligned}
f^{\left(\alpha_{1}\right)}(0)\left(a_{1} \gamma_{1}^{\alpha_{1}}+a_{2} \gamma_{2}^{\alpha_{1}}+\ldots+a_{n} \gamma_{n}^{\alpha_{1}}\right) & =0 \\
f^{\left(\alpha_{2}\right)}(0)\left(a_{1} \gamma_{1}^{\alpha_{2}}+a_{2} \gamma_{2}^{\alpha_{2}}+\ldots+a_{n} \gamma_{n}^{\alpha_{2}}\right) & =0 \\
\ldots & =0 \\
f^{\left(\alpha_{n-1}\right)}(0)\left(a_{1} \gamma_{1}^{\alpha_{n-1}}+a_{2} \gamma_{2}^{\alpha_{n-1}}+\ldots+a_{n} \gamma_{n}^{\alpha_{n-1}}\right) & =0 .
\end{aligned}
$$

So, in the case that $f^{(\alpha)}(0) \neq 0$ for some $\alpha \neq \alpha_{j}, j=1, \ldots, n-1$, we would also have

$$
a_{1} \gamma_{1}^{\alpha}+a_{2} \gamma_{2}^{\alpha}+\ldots+a_{n} \gamma_{n}^{\alpha}=0
$$

which would lead to $a_{1}=a_{2}=\ldots=a_{n}=0$.

Note that the proposition above is related to [12, Proposition 5], which shows that, under the condition of positive coefficients $a_{j}>0$, the only solution of the functional equation (3.1) that is analytic at 0 is the trivial solution $f \equiv 0$.

Example 3.3 Consider the functional equation $3 f(s)-4 f(2 s)+f(5 s)=0$ with $s \in \mathbb{C}$. By Proposition 2.9, we know that the functions of the form $f_{w_{0}}(s)=$ $\left\{\begin{array}{ll}e^{w_{0} \log _{\theta} s} & \text { if } s \neq 0 \\ 0 & \text { if } s=0\end{array}\right.$, where $w_{0}$ is a zero of the exponential polynomial given by $P(s)=3-4 \cdot 2^{s}+5^{s}$, are solutions analytic on $\mathbb{C} \backslash R_{\theta}$ of such an equation. As $P(0)=0$, the constant solution $f_{0}(s)=1$ is a particular case, but $P(s)$ has infinitely many zeros (all of them lie in a vertical strip, see for example [9]) which provide other solutions $f_{w_{0}}(s)$ which are not polynomials. Now, by Proposition 3.1, the only solutions of such an equation which are analytic at 0 
are the polynomials of, at most, 2 nonzero terms. In fact, it is easy to check that the polynomials of the form $f(s)=A+B s$, with $A, B \in \mathbb{C}$, are solutions of such a functional equation.

Secondly, under the hypothesis of analyticity on an annulus centered at 0 , we shall obtain solutions which are meromorphic on such a set. Recall that a function $f$ is meromorphic on a set $S$ if $f$ is analytic on $S$ except possibly for poles [2].

Proposition 3.4 Let $f(s)$ be a complex solution of the functional equation

$$
a_{1} f\left(\gamma_{1} s\right)+a_{2} f\left(\gamma_{2} s\right)+\ldots+a_{n} f\left(\gamma_{n} s\right)=0, s \in S,
$$

where $n \in \mathbb{N}, n \geq 2,\left\{\gamma_{1}, \gamma_{2}, \ldots, \gamma_{n}\right\}$ is a set of distinct positive numbers, $a_{j} \in \mathbb{C} \backslash\{0\}$ for each $j=1,2, \ldots, n$ and $S$ is a certain open set that does not contain 0 . If $f(s)$ is analytic on some annulus $\{s \in \mathbb{C}: r<|s|<R\}$ with $0 \leq r<R \leq \infty$, then it is a meromorphic function, analytic on $\mathbb{C} \backslash\{0\}$, whose Laurent expansion about 0 has, at most, $n-1$ nonzero terms.

Proof. Assume the existence of a solution of (3.3) which is analytic on some annulus of the form $D=\{s \in \mathbb{C}: r<|s|<R\}$, say $f(s)$. This means that $f(s)$ has a Laurent series on $D$ of the form $f(s)=\sum_{k=-\infty}^{\infty} b_{k} s^{k} \forall s \in D$. Hence

$$
\begin{aligned}
& a_{1} f\left(\gamma_{1} s\right)+a_{2} f\left(\gamma_{2} s\right)+\ldots+a_{n} f\left(\gamma_{n} s\right)= \\
& a_{1} \sum_{k=-\infty}^{\infty} b_{k} \gamma_{1}^{k} s^{k}+a_{2} \sum_{k=-\infty}^{\infty} b_{k} \gamma_{2}^{k} s^{k}+\ldots+a_{n} \sum_{k=-\infty}^{\infty} b_{k} \gamma_{n}^{k} s^{k}= \\
& \ldots+\left(a_{1} b_{-1} \gamma_{1}^{-1}+a_{2} b_{-1} \gamma_{2}^{-1}+\ldots+a_{n} b_{-1} \gamma_{n}^{-1}\right) \frac{1}{s}+\left(a_{1} b_{0}+a_{2} b_{0}+\ldots+a_{n} b_{0}\right) \\
& +\left(a_{1} b_{1} \gamma_{1}+a_{2} b_{1} \gamma_{2}+\ldots+a_{n} b_{1} \gamma_{n}\right) s+\ldots=0
\end{aligned}
$$

for all $s$ in a set that contains $D$ and is included in $S$. This yields that

$$
\begin{aligned}
\ldots & =0 \\
b_{-1}\left(a_{1} \gamma_{1}^{-1}+a_{2} \gamma_{2}^{-1}+\ldots+a_{n} \gamma_{n}^{-1}\right) & =0 \\
b_{0}\left(a_{1}+a_{2}+\ldots+a_{n}\right) & =0 \\
b_{1}\left(a_{1} \gamma_{1}+a_{2} \gamma_{2}+\ldots+a_{n} \gamma_{n}\right) & =0 \\
\ldots & =0 .
\end{aligned}
$$

If we took $\mathbf{a}=\left(a_{1}, a_{2}, \ldots\right)^{t}$ as the vector of the unknowns, it would lead to a matrix equation of the form $M \mathbf{a}=\mathbf{0}$, where

$$
M=\left(\begin{array}{cccc}
\vdots & \vdots & \vdots & \vdots \\
b_{-1} \gamma_{1}^{-1} & b_{-1} \gamma_{2}^{-1} & \ldots & b_{-1} \gamma_{n}^{-1} \\
b_{0} & b_{0} & \ldots & b_{0} \\
b_{1} \gamma_{1} & b_{1} \gamma_{2} & \ldots & b_{1} \gamma_{n} \\
\vdots & \ldots & \ddots & \vdots
\end{array}\right)
$$


Under the hypothesis of the existence of at least $n$ values of the set given by $B=\left\{\ldots, b_{-1}, b_{0}, b_{1}, \ldots\right\}$ which are nonzero, it is clear that $\operatorname{rank} M=n$ (it leads to a matrix of the same form as the generalized Vandermonde matrix in the proof of Proposition 3.1, see also [13, Theorem 1]) or, equivalently, the trivial solution $a_{1}=a_{2}=\ldots=a_{n}=0$ is the only solution of this system, which is a contradiction. Consequently, 0 is not an essential singularity of $f(s)$, i.e. $f(s)$ is a meromorphic function of the form $\sum_{k=-\infty}^{\infty} b_{k} s^{k}$, where the cardinal of the set $B$ is less than $n$, which proves the result.

Remark 3.5 As in Remark 3.2, from the proof of the proposition above it is also deduced that if $P(s)=s^{\alpha_{1}}+s^{\alpha_{2}}+\ldots+s^{\alpha_{n-1}}$, with $\alpha_{1}<\alpha_{2}<\ldots<\alpha_{n-1}$, is a meromorphic function of $n-1$ terms which is solution of the functional equation (3.3), then any solution of such an equation is of the form

$$
f(s)=c_{1} s^{\alpha_{1}}+c_{2} s^{\alpha_{2}}+\ldots+c_{n-1} s^{\alpha_{n-1}},
$$

with $c_{1}, c_{2}, \ldots, c_{n-1} \in \mathbb{C}$. As a consequence, in this case we would have the following result: if $f(s)$ is a solution of $n-1$ terms of the functional equation (defined on a certain set $S$ that does not contain 0 ), then such an equation (extended onto $S \cup\{0\}$ ) has solutions analytic at 0 if and only if some of the $n-1$ terms of $f(s)$ is of the form $a_{j} s^{j}$ with $j=0,1, \ldots$.

Example 3.6 Consider the functional equation $f\left(\frac{1}{5} s\right)-4 f\left(\frac{1}{2} s\right)+3 f(s)=0$ with $s \in \mathbb{C} \backslash\{0\}$. By Proposition 3.4, the only non-trivial solutions of such an equation which are analytic on the annulus $\{s \in \mathbb{C}: 0<|s|<R\}$, with $R>0$, are the meromorphic functions of, at most, 2 nonzero terms. In fact, it is easy to check that $f(s)=A+\frac{B}{s}$, with $A, B \in \mathbb{C}$, is a solution of such an equation which is not of the form of Proposition 2.9. Moreover, the constant polynomials $P(s)=A$, with $A \in \mathbb{C}$, are solutions of the functional equation extended onto the whole $\mathbb{C}$.

Finally, inspired by the proof of Proposition 3.4, we will prove the following result.

Proposition 3.7 Consider $\left\{\gamma_{1}, \gamma_{2}, \ldots, \gamma_{j}, \ldots\right\}$ a set of distinct positive numbers and $S \subset \mathbb{C}$ an open set that does not contain 0 . Let $f(s)$ be a meromorphic function whose Laurent expansion about 0 has $n \geq 2$ nonzero terms, and let $m$ be an integer number greater than $n$. Then $f(s)$ is a solution, which is analytic on $\mathbb{C} \backslash\{0\}$, of infinitely many functional equations of the form

$$
a_{1} f\left(\gamma_{1} s\right)+a_{2} f\left(\gamma_{2} s\right)+\ldots+a_{m} f\left(\gamma_{m} s\right), s \in S,
$$

for some $a_{j} \in \mathbb{C} \backslash\{0\}, j=1,2, \ldots, m$.

Proof. Assume that $f(s)$ has a Laurent series, on the annulus $D=\{s \in \mathbb{C}$ : $|s|>0\}$, of the form $f(s)=\sum_{k=-\infty}^{\infty} b_{k} s^{k} \forall s \in D$, where the cardinal of the 
set $B=\left\{b_{k_{1}}, b_{k_{2}}, \ldots, b_{k_{n}}\right\}, k_{j} \in \mathbb{Z}$, of nonzero coefficients is equal to $n$. Let $m \geq n+1$, then

$$
\begin{aligned}
& a_{1} f\left(\gamma_{1} s\right)+a_{2} f\left(\gamma_{2} s\right)+\ldots+a_{m} f\left(\gamma_{m} s\right)= \\
& a_{1} \sum_{j=1}^{n} b_{k_{j}} \gamma_{1}^{k_{j}} s^{k_{j}}+a_{2} \sum_{j=1}^{n} b_{k_{j}} \gamma_{2}^{k_{j}} s^{k_{j}}+\ldots+a_{m} \sum_{j=1}^{n} b_{k_{j}} \gamma_{m}^{k_{j}} s^{k_{j}}= \\
& \quad\left(a_{1} b_{k_{1}} \gamma_{1}^{k_{1}}+a_{2} b_{k_{1}} \gamma_{2}^{k_{1}}+\ldots+a_{m} b_{k_{1}} \gamma_{m}^{k_{1}}\right) s^{k_{1}}+\ldots+ \\
& \quad+\left(a_{1} b_{k_{n}} \gamma_{1}^{k_{n}}+a_{2} b_{k_{n}} \gamma_{2}^{k_{n}}+\ldots+a_{m} b_{k_{n}} \gamma_{m}^{k_{n}}\right) s^{k_{n}}=0
\end{aligned}
$$

for all $s$ in a set that contains $D$ and is included in $S$. This yields that

$$
\begin{aligned}
b_{k_{1}}\left(a_{1} \gamma_{1}^{k_{1}}+a_{2} \gamma_{2}^{k_{1}}+\ldots+a_{m} \gamma_{m}^{k_{1}}\right)= & 0 \\
\ldots & 0 \\
b_{k_{n}}\left(a_{1} \gamma_{1}^{k_{n}}+a_{2} \gamma_{2}^{k_{n}}+\ldots+a_{m} \gamma_{m}^{k_{n}}\right)= & 0
\end{aligned}
$$

If we took $\mathbf{a}=\left(a_{1}, a_{2}, \ldots, a_{m}\right)^{t}$ as the vector of the unknowns, it would lead to a system of the form $M \mathbf{a}=\mathbf{0}$, where

$$
M=\left(\begin{array}{cccc}
\gamma_{1}^{k_{1}} & \gamma_{2}^{k_{1}} & \ldots & \gamma_{m}^{k_{1}} \\
\vdots & \vdots & \vdots & \vdots \\
\gamma_{1}^{k_{n}} & \gamma_{2}^{k_{n}} & \cdots & \gamma_{m}^{k_{n}}
\end{array}\right)
$$

satisfies $\operatorname{rank} M=n$ (it leads to a matrix of the same form as the generalized Vandermonde matrix in the proof of Proposition 3.1). If $m \geq n+1$, it is clear that this system has infinitely many solutions, which proves the result.

\section{References}

[1] Almira, J.M.; Abu-Helaiel, Kh. F.: On solutions of $f(x)+f\left(a_{1} x\right)+$ $\ldots+f\left(a_{N} x\right)=0$ and related equations, Ann. Tiberiu Popoviciu Semin. Funct. Equ. Approx. Convexity. 9 (2011), 3-17.

[2] Ash, R.B.: Complex variables, Academic Press, London, 1971.

[3] Besicovitch, A.S.: Almost periodic functions, Dover, New York, 1954.

[4] Bohr, H.: Zur Theorie der fastperiodischen Funktionen. (German) III. Dirichletentwicklung analytischer Funktionen, Acta Math. 47 (3) (1926), 237-281.

[5] Bohr, H.: Almost periodic functions, Chelsea, New York, 1951.

[6] Corduneanu, C.: Almost Periodic Functions, Interscience publishers, New York, London, Sydney, Toronto, 1968. 
[7] Favorov, S. Yu.: Zeros of Holomorphic Almost-Periodic Functions, Zeros of holomorphic almost periodic functions. J. Anal. Math. 84 (2001), 51-66.

[8] Jessen, B.: Some aspects of the theory of almost periodic functions, in Proc. Internat. Congress Mathematicians Amsterdam, 1954, Vol. 1, NorthHolland, 1954, pp. 304-351.

[9] Mas, A.; Sepulcre, J.M.: The projections of the zeros of exponential polynomials with complex frequencies, Colloq. Math. 158 (1) (2019), 91102.

[10] Mora, G.: A note on the functional equation $F(z)+F(2 z)+\cdots+F(n z)=$ 0, J. Math. Anal. Appl. 340 (2008), 466-475.

[11] Mora, G.; Sepulcre, J.M.: The zeros of Riemann zeta partial sums yield solutions to $f(x)+f(2 x)+\ldots+f(n x)=0$, Mediterr. J. Math. 10 (2013), 3, 1221-1232.

[12] Sepulcre, J.M.; Vidal, T.: On the analytic solutions of the functional equations $w_{1} f\left(a_{1} z\right)+w_{2} f\left(a_{2} z\right)+\ldots+w_{n} f\left(a_{n} z\right)=0$, Mediterr. J. Math., 12 (2015), 667-678.

[13] Yang, S.J.; Wu, H.Z.; Zhang, Q.B.: Generalization of Vandermonde determinants, Linear Algebra and its Applications, 336 (2001), 201-204. 